\title{
Infective Endocarditis Associated with Mitral Valve Prolapse in a Patient with Klinefelter Syndrome
}

\author{
Yasushi Ueki ${ }^{1}$, Atsushi Izawa ${ }^{1}$, Souichiro Ebisawa ${ }^{1}$, Hirohiko Motoki ${ }^{1}$, Yusuke Miyashita ${ }^{1}$, \\ Takeshi Tomita ${ }^{1}$, Jun Koyama ${ }^{1}$, Tamaki Takano ${ }^{2}$, Jun Amano ${ }^{2}$ and Uichi Ikeda ${ }^{1}$
}

\begin{abstract}
We herein report a case of infective endocarditis associated with mitral valve prolapse (MVP) in a 34-yearold man with Klinefelter syndrome. The patient was admitted with a fever and headache that had persisted for three weeks. Repeated blood cultures showed growth of Streptococcus oralis. Echocardiography demonstrated severe mitral regurgitation with a large vegetation attached to the prolapsed anterior leaflet. Surgical plasty of the mitral valve was performed because the vegetation measured over $10 \mathrm{~mm}$ in diameter and there was a risk of recurrence of embolic complications. This case demonstrates the link between MVP and Klinefelter syndrome and highlights the importance of performing cardiovascular screening and preventing endocarditis.
\end{abstract}

Key words: infective endocarditis, mitral valve prolapse, Klinefelter syndrome, echocardiography

(Intern Med 53: 969-972, 2014)

(DOI: 10.2169/internalmedicine.53.1748)

\section{Introduction}

Prolapse of the mitral valve has been identified in patients with hereditary and connective tissue disorders, including Marfan syndrome, Ehlers-Danlos syndrome and pseudoxanthoma elasticum, as well as patients with Klinefelter syndrome, the most common genetic cause of male infertility. We herein report a rare complication of infective endocarditis of a prolapsed mitral valve in a patient with Klinefelter syndrome.

\section{Case Report}

A 34-year-old man was admitted to our hospital with a fever and headache lasting for three weeks despite the administration of primary treatment with antibiotics. The patient was diagnosed with a chromosomal abnormality of Klinefelter syndrome after undergoing in vitro fertilization therapy. In his past clinical course, he had received no diagnoses of heart murmurs, diabetes mellitus, obesity or mental retardation. His bone mineral density and hormonal abnor- malities were not examined precisely, and he received no testosterone substitution therapy. He had no family history of genetic disease or valvular heart disease. On the second day after admission, two sets of his blood cultures showed growth of Streptococcus oralis. Echocardiography revealed severe regurgitation of the mitral valve associated with a vegetation on the anterior leaflet. The patient was diagnosed with infective endocarditis of the mitral valve and transferred to our department.

On a physical examination, the patient's body height was $180 \mathrm{~cm}$, his body weight was $57 \mathrm{~kg}$ and his temperature was $37.6^{\circ} \mathrm{C}$. An oral examination revealed many cavities in the teeth. No Osler's nodes or Janeway's lesions were observed. A grade 2/6 systolic regurgitant murmur was heard at the apex. No wrist signs or thumb signs suggestive of Marfan syndrome were noted. A chest radiograph showed no cardiomegaly. Echocardiography (Fig. 1) revealed severe regurgitation of the mitral valve with prolapse of the anterior leaflet, and the left ventricular ejection fraction was $77 \%$. A vegetation associated with endocarditis (diameter, $13 \mathrm{~mm}$ ) was attached to the anterior mitral leaflet, as confirmed on transesophageal echocardiography (Fig. 2). Eleva-

${ }^{1}$ Department of Cardiovascular Medicine, Shinshu University School of Medicine, Japan and ${ }^{2}$ Department of Cardiovascular Surgery, Shinshu University School of Medicine, Japan

Received for publication September 12, 2013; Accepted for publication November 24, 2013

Correspondence to Dr. Atsushi Izawa, izawa611@shinshu-u.ac.jp 

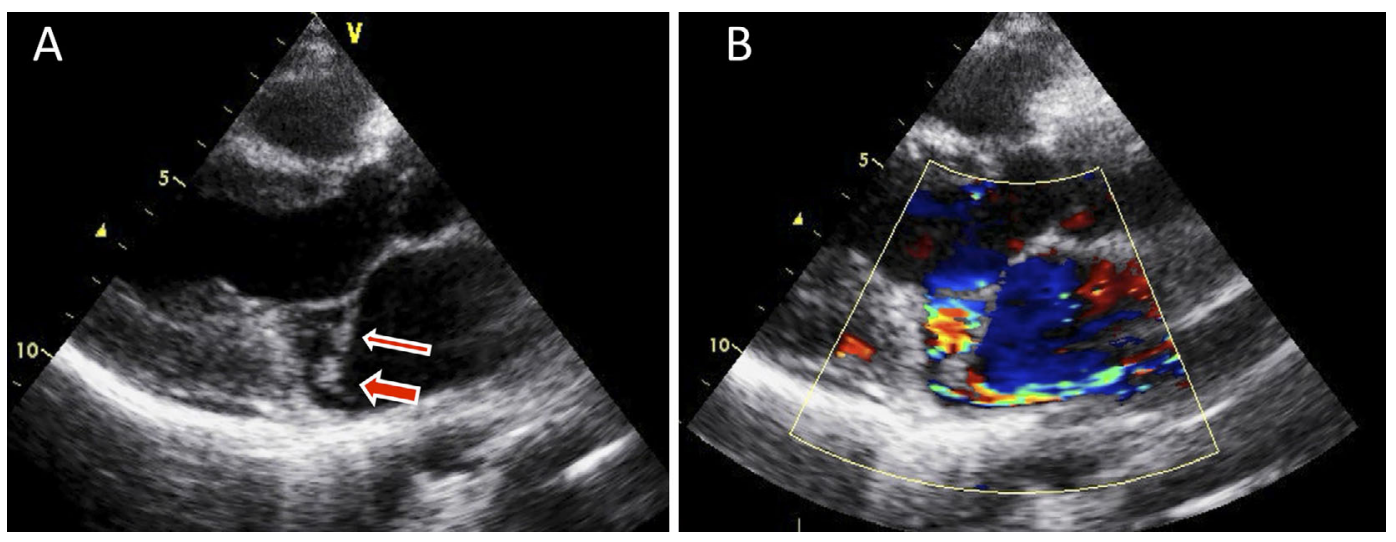

Figure 1. Transthoracic echocardiography. A) Prolapse of the anterior mitral leaflet (thin arrow). The diameter of the vegetation is $\mathbf{1 3} \mathbf{~ m m}$ (thick arrow); B) Mitral insufficiency showing a regurgitated flow along the posterior wall of the left atrium.
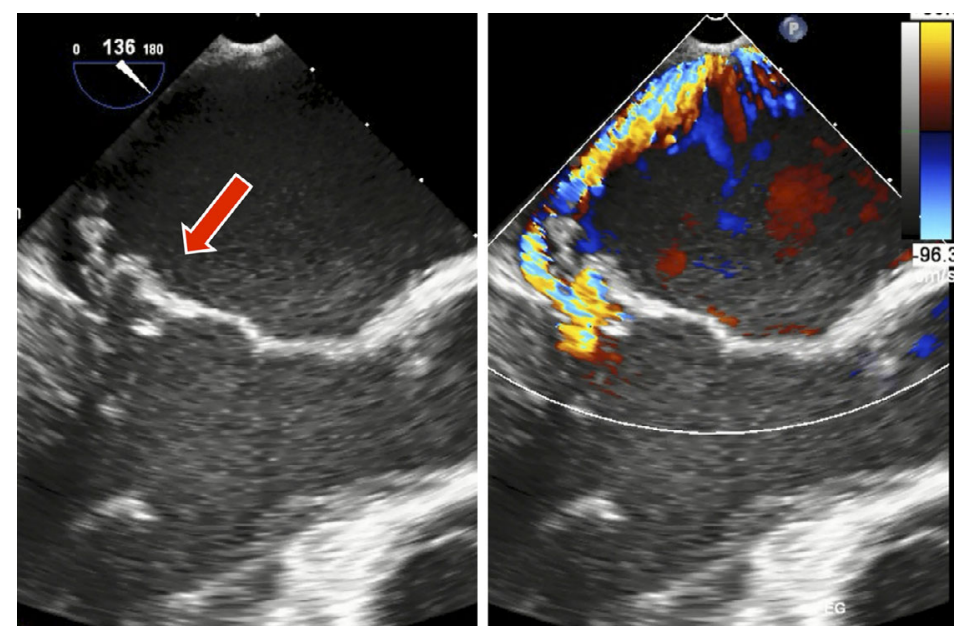

Figure 2. Transesophageal echocardiography. Prolapse of the anterior mitral leaflet (arrow) and a mitral regurgitation flow directed to the posterior wall of the left atrium. A vegetation is attached to the anterior mitral leaflet.

tion of the C-reactive protein $(2.83 \mathrm{mg} / \mathrm{dL})$ and procalcitonin $(1.9 \mathrm{ng} / \mathrm{mL})$ levels suggested systemic inflammation.

The intravenous daily administration of ampicillin (12 g) and gentamicin $(120 \mathrm{mg})$ was initiated, which reduced the patient's fever. On the second day after admission, enhanced computed tomography (CT) and brain magnetic resonance imaging (MRI) (Fig. 3) showed embolic infarction of the spleen and cerebrum. Due to these embolic complications and the fact that the vegetation measured over $1 \mathrm{~cm}$ in diameter, surgical plasty of the mitral valve was performed on day 22 after admission, according to the Japanese Circulation Society guidelines for the prevention and treatment of infective endocarditis. The vegetation was attached to a ruptured A2 strut chorda, which was removed and reconstructed with artificial chordae. Tissue sections of the mitral valve revealed a myxomatous valve structure with surface accumulation of fibrins and necrotic tissue (Fig. 4). These findings were conventional and compatible with a diagnosis of myxomatous degeneration accompanied by infective endocarditis. During the patient's postoperative treatment, he suf- fered from skin eruptions induced by ampicillin and/or gentamicin; these agents were thus switched to ceftriaxone and vancomycin. He was discharged on day 79 with no other complications.

\section{Discussion}

The most common sex chromosome abnormality, Klinefelter syndrome, is defined as the presence of at least one $\mathrm{Y}$ chromosome and two or more $\mathrm{X}$ chromosomes and is characterized by gynecomastia, azoospermia and small testes, resulting in male infertility. Although the estimated prevalence of Klinefelter syndrome is approximately 1 in 600 male newborns (1), only one-fourth of adult men are diagnosed due to the discrete nature of the symptoms.

Mitral valve prolapse (MVP) is a common cause of mitral regurgitation, with an estimated prevalence of $2.4 \%$ in the general population according to the Framingham Heart Study (2). Fricke et al. reported 12 patients with MVP and three patients with mitral regurgitation among 22 patients 

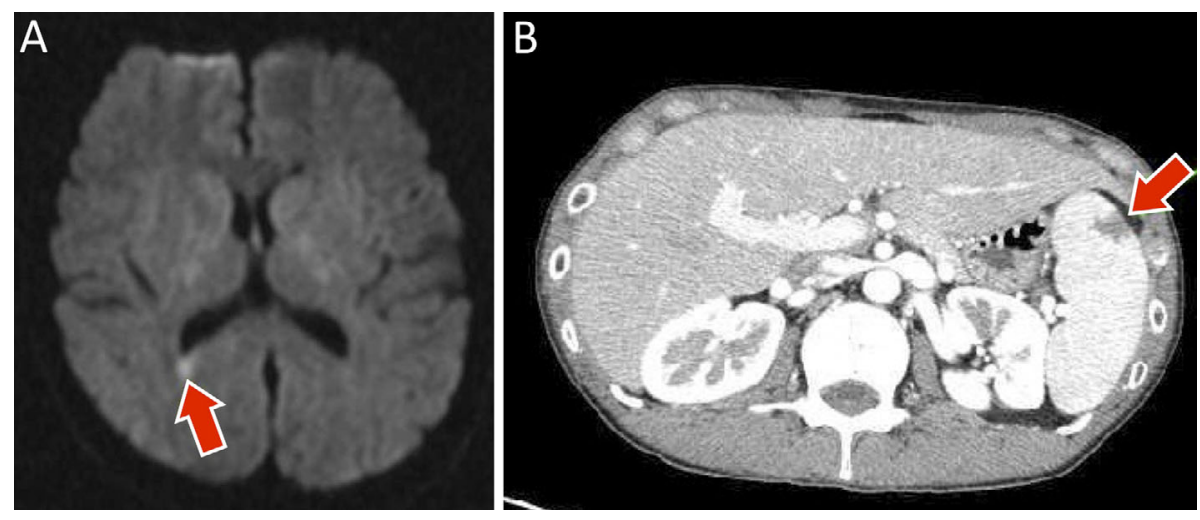

Figure 3. A) A magnetic resonance image of the brain: High signal intensity in the back right side of the posterior horn on a diffusion image (arrow). B) An enhanced computed tomography abdominal scan: An enhancement defect in the spleen, suggesting an embolized area (arrow).
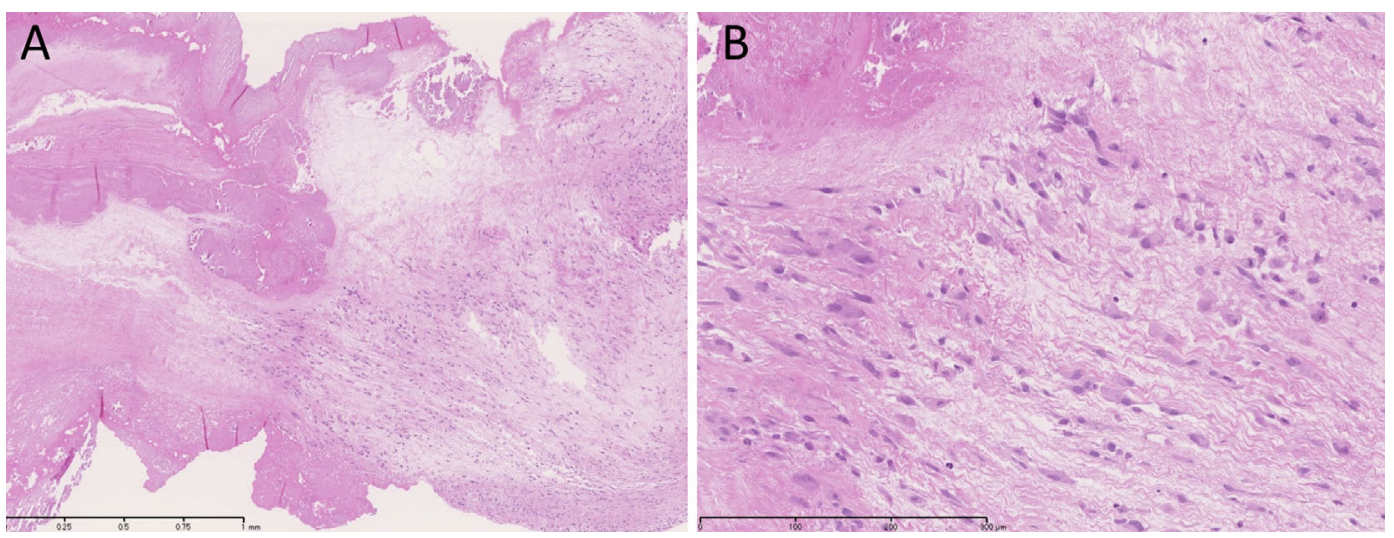

Figure 4. Tissue sections treated with Hematoxylin and Eosin staining of the mitral leaflet. Fibrous hypertrophy and myxomatous degeneration are observed in association with the accumulation of fibrins and necrotic tissue on the surface (A: bar=1 mm; B: bar=300 $\mu \mathrm{m}$ ).

with Klinefelter syndrome $(3,4)$. A case of Klinefelter syndrome with acute regurgitation of the mitral valve caused by myxomatous degeneration of the valve and ruptured chordae, suggesting the involvement of the subvalvular apparatus, has also been reported (5). Clinicians should therefore pay attention to possible abnormalities of the mitral valve structure in patients with Klinefelter syndrome. On the other hand, in one study, no cases of MVP were noted among 69 patients with Klinefelter syndrome in comparison with 48 age-matched controls (6). In addition, in another study, three (12\%) of 25 patients with Klinefelter syndrome exhibited small mitral valve regurgitation; however, none of these patients had primary mitral valve disease, including MVP (7). To date, the clinical findings are insufficient to determine the exact prevalence of and pathological association between Klinefelter syndrome and MVP. The accumulation of cases and large-scale cohort studies is therefore required to clarify the specific pathology and causal association between Klinefelter syndrome and MVP.

MVP is more prevalent in patients with connective tissue disorders, including Marfan syndrome (8), Ehlers-Danlos syndrome (9), osteogenesis imperfecta (10) and pseudoxanthoma elasticum (11). Bastianon et al. reported that 12 of 46 patients with Turner syndrome had MVP (12). The risk of aortic dissection is also significantly increased in patients with Turner syndrome $(13,14)$. Therefore, sex chromosome disorders may potentially induce vulnerability of connective tissue, which plays a key role in MVP, as shown in this case and other reports of Klinefelter syndrome (5). Interestingly, a locus on chromosome $\mathrm{X}, \mathrm{Xq} 28$, has been found to cosegregate with that of $\mathrm{X}$-linked myxomatous valvular dystrophy $(15,16)$. In addition, mutations in the filamin A gene were recently identified in a large French family with Xlinked myxomatous valvular dystrophy $(17,18)$.

Klinefelter syndrome is the most common sex chromosome abnormality; however, many adult patients remain undiagnosed. This report highlights the importance of making a genetic diagnosis and screening for valvular diseases in patients with Klinefelter syndrome and other diseases associated with connective tissue vulnerability. Conversely, patients with a diagnosis of MVP may require a differential diagnosis of Klinefelter syndrome or other connective tissue diseases. Although the incidence of infectious endocarditis is very low in patients with MVP, primary prevention of endocarditis is warranted when a patient with Klinefelter syndrome exhibits MVP. 
The authors state that they have no Conflict of Interest (COI).

\section{References}

1. Wikstrom AM, Dunkel L. Klinefelter syndrome. Best Pract Res Clin Endocrinol Metab 25: 239-250, 2011.

2. Freed LA, Benjamin EJ, Levy D, et al. Mitral valve prolapse in the general population: the benign nature of echocardiographic features in the Framingham Heart Study. J Am Coll Cardiol 40: 1298-1304, 2002.

3. Fricke GR, Mattern HJ, Schweikert HU, Schwanitz G. Klinefelter's syndrome and mitral valve prolapse: an echocardiographic study in twenty-two patients. Biomed Pharmacother 38: 88-97, 1984.

4. Fricke GR, Mattern HJ, Schweikert HU. Mitral valve prolapse in Klinefelter syndrome. Lancet 26: 1414, 1981.

5. Katsumata T, Tomizawa Y, Okoshi T, et al. A case of Klinefelter's syndrome with acute mitral regurgitation caused by idiopathic chordal rupture. Kyobu Geka 44: 867-870, 1991 (in Japanese, Abstract in English).

6. Pasquali D, Arcopinto M, Renzullo A, et al. Cardiovascular abnormalities in Klinefelter syndrome. Int J Cardiol 168: 754-759, 2013.

7. Andersen NH, Bojesen A, Kristensen $\mathrm{K}$, et al. Left ventricular dysfunction in Klinefelter syndrome is associated to insulin resistance, abdominal adiposity and hypogonadism. Clin Endocrinol (Oxf) 69: 785-791, 2008.

8. Read RC, Thal AP, Wendt VE. Symptomatic valvular myxomatous transformation (the floppy valve syndrome): a possible forme fruste of the Marfan syndrome. Circulation 32: 897-910, 1965.

9. Takano H, Miyamoto Y, Sawa Y, et al. Successful mitral valve replacement in a patient with Ehlers-Danlos syndrome type IV. Ann Thorac Surg 80: 320-322, 2005.

10. Glesby MJ, Pyeritz RE. Association of mitral valve prolapse and systemic abnormalities of connective tissue. A phenotypic continuum. JAMA 262: 523-528, 1989.

11. Rubegni P, Mondillo S, De Aloe G, Aqricola E, Bardelli AM, Fimiani M. Mitral valve prolapse in healthy relatives of patients with familial Pseudoxanthoma elasticum. Am J Cardiol 85: 12681271, 2000.

12. Bastianon V, Pasquino AM, Giglioni E, et al. Mitral valve prolapse in Turner syndrome. Eur J Pediatr 148: 533-534, 1989.

13. Sybert VP. Cardiovascular malformations and complications in Turner syndrome. Pediatrics 101: E11, 1998.

14. Lin AE, Lippe BM, Geffner ME, et al. Aortic dilation, dissection, and rupture in patients with Turner syndrome. J Pediatr 109: 820826, 1986.

15. Kyndt F, Schott JJ, Trochu JN, et al. Mapping of X-linked myxomatous valvular dystrophy to chromosome Xq28. Am J Hum Genet 62: 627-632, 1998.

16. Trochu JN, Kyndt F, Schott JJ, et al. Clinical characteristics of a familial inherited myxomatous valvular dystrophy mapped to Xq28. J Am Coll Cardiol 35: 1890-1897, 2000.

17. Kyndt F, Gueffet JP, Probst V, et al. Mutations in the gene encoding filamin A as a cause for familial cardiac valvular dystrophy. Circulation 115: 40-49, 2007.

18. Lardeux A, Kyndt F, Lecointe S, et al. Filamin-a-related myxomatous mitral valve dystrophy: genetic, echocardiographic and functional aspects. J Cardiovasc Transl Res 4: 748-756, 2011.

(C) 2014 The Japanese Society of Internal Medicine http://www.naika.or.jp/imonline/index.html 